\title{
Are N-methyl groups of Tetramethylurea (TMU) Hydrophobic? A composition and temperature-dependent fluorescence spectroscopic investigation of TMU/water binary mixtures
}

\author{
SANDIPA INDRA and RANJIT BISWAS* \\ Department of Chemical, Biological and Macromolecular Sciences, S N Bose National Centre for Basic \\ Sciences, Block J D, Sector III, Salt Lake, Kolkata 700 098, India \\ e-mail: ranjit@bose.res.in
}

MS received 1 December 2015; revised 12 February 2016; accepted 18 February 2016

\begin{abstract}
This paper reports results from temperature and composition dependent steady state UV-Visible absorption and fluorescence measurements and time-resolved fluorescence experiments, using a dipolar probe coumarin 153 (C153), in TMU/water binary mixtures. Both steady state and time-resolved spectroscopic data indicate, much like in water/alcohol mixtures, TMU-induced structural stiffening and transition of the tetrahedral H-bond network of water. The structural transition referred to above means cosolvent-induced transition of three dimensional tetrahedral H-bond network to two dimensional zig-zag chain-like structure often found in alcohols. A comparison to the results obtained for aqueous alcohol solutions suggests that the cosolvent concentration at which the structural transition occurs depends both on the polarity of the cosolvent and the size of the cosolvent molecules. UV-Visible absorption measurements reveal aggregation among TMU molecules at lower TMU concentration which shows a temperature maximum. In addition, red edge excitation effects have been observed at very dilute TMU concentration suggesting distribution of C153 among heterogeneous environments. All these results indicate hydrophobic interaction-induced aggregation of TMU in dilute aqueous solutions which corroborate well with the existing simulation observation.
\end{abstract}

Keywords. Tetramethylurea; aqueous solution; hydrophobicity; fluorescence study.

\section{Introduction}

Recent study ${ }^{1}$ of thermodynamic properties of aqueous solutions of amphiphiles suggested that methyl group, which is known to be hydrophobic in nature, may not always show hydrophobic character. The hydrophobic nature of methyl group depends on the atom to which it is attached. The study also indicated that methyl groups attached to electronegative nitrogen atoms show hydrophilicity rather than hydrophobicity. Tetramethylurea (TMU, $\left.\left(\left(\mathrm{CH}_{3}\right)_{2} \mathrm{~N}\right)_{2} \mathrm{CO}\right)$ is an example of this type of molecule which possesses amphiphilic character as well as $\mathrm{N}$-methyl $\left(-\mathrm{N}-\mathrm{CH}_{3}\right)$ groups. Another important characteristic of TMU is the strong protein denaturing ability. ${ }^{2,3}$ Interestingly, computer simulation studies ${ }^{4-6}$ have shown TMU aggregation via hydrophobic interactions among the methyl $\left(-\mathrm{CH}_{3}\right)$ groups in aqueous solutions at very low concentrations. Disagreement among different studies stimulates further research in this area.

Study of binary mixtures of water with other cosolvents, specially, those which can interact with water

*For correspondence via both hydrogen bond donor and acceptor sites such as alcohols, has long been a matter of discussion. ${ }^{7-27}$ Researchers have found that the tetrahedral hydrogen bonding network of water becomes stronger in presence of small amount of alcohols due to their accommodation into the tetrahedral cavity. This three dimensional tetrahedral $\mathrm{H}$-bond network structure of water gets disrupted to two dimensional zig-zag chainlike structure at comparatively larger alcohol concentrations where the tetrahedral network of water can no longer accommodate alcohol molecules..$^{17,18,20}$ This effect is more pronounced while moving from small alcohols like ethanol $(\mathrm{EtOH})$ to larger alcohols like tertiary butanol (TBA) because hydrophobic interaction plays a critical role in regulating the structural arrangement of both the specie in a given binary mixture. ${ }^{18}$ Consequently, dynamical properties of aqueous solutions exhibit significant changes in presence of co-solvents.

Several experimental studies such as dielectric relaxation, ${ }^{28}$ femtosecond IR, ${ }^{29,30}$ and ultrafast optical Kerr effect ${ }^{31}$ measurements revealed that in aqueous solutions of TMU, the reorientational dynamics of water in hydrophobic hydration shell undergoes substantial 
retardation than that in bulk water. Subsequent computer simulation studies ${ }^{32-34}$ have suggested that slower $\mathrm{H}$-bond exchange near the hydrophobic groups is the key for retarded reorientational dynamics of water. We have also observed the slowing down of water-water H-bond persistence time in presence of TMU in water. ${ }^{6}$ In concentrated TMU solutions, the lengthening of H-bond dynamical timescales due to solute-induced confinement of water is reminiscent of water in explicit confined environment. ${ }^{35,36}$

Here, we explore whether the hydrophobic part containing four $\mathrm{N}$-methyl groups of TMU acts in the similar manner in aqueous solutions as we had observed before in aqueous alcoholic solutions. ${ }^{17-20}$ In this paper, we have investigated various compositions of TMU in water at different temperatures by UVVisible absorption and fluorescence spectroscopic measurements. Steady state absorption and fluorescence emission of a nonreactive probe coumarin 153 (C153) in TMU/water binary mixtures have provided important information regarding the structural change in the three dimensional H-bonding network of water upon addition of TMU. As we know, solution dynamics is intimately related to the solution structure, TMU-induced modification of bulk water structure will alter solution dynamics. Time-resolved fluorescence anisotropy measurements have been carried out to probe the solute-solvent coupling in this microscopically heterogeneous system. Fluorescence decay measurements, on the other hand, provide a qualitative idea about the effects of modified solution structure on solvent relaxation. Chemical structure of TMU is shown in scheme 1 along with three other co-solvents considered in the present study.

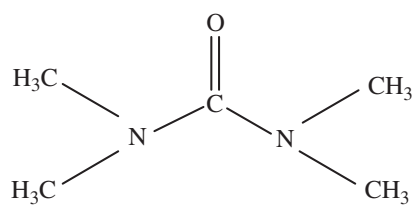

(a)

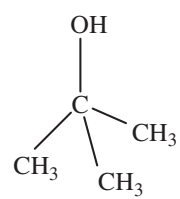

(c)

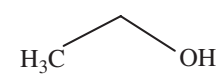

(b)

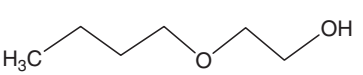

(d)
Scheme 1. Chemical Structures of (a) Tetramethylurea (TMU), (b) Ethanol (EtOH), (c) Tertiary butanol (TBA), (d) 2-butoxyethanol (BE).

\section{Experimental Section}

\subsection{Sample Preparation}

Laser grade C153 and TMU, purchased from Exciton and Sigma-Aldrich, respectively, were used as received. Deionized (Millipore) water was used for preparing different compositions of TMU/water mixtures. Binary solvent mixtures were prepared by adding measured amounts of TMU into measured amounts of water in a volumetric flask, followed by a gentle shaking of the solution for a few minutes. Proper caution was exercised to ensure a complete transfer of either TMU or water while preparing the solutions particularly at the low mole fraction regime. Subsequently, a few $\mu \mathrm{L}$ of freshly prepared solution of C153 in heptane was poured into a quartz cuvette of optical path length $1 \mathrm{~cm}$. The nonpolar solvent was then evaporated off by gently blowing hot air around the outer surface of the cuvette. Subsequenly, 3-4 mL of aliquot was added to the cuvette and stirred the solution for sometimes to ensure complete dissolution of the $\mathrm{C} 153$ grains. Note that the concentration of $\mathrm{C} 153$ was maintained at $\leq$ $10^{-5} \mathrm{M}$ in all TMU mole fractions studied here.

\subsection{Data Collection and Analysis for Steady-state and Time-resolved Fluorescence Measurements}

Absorption and fluorescence emission spectra of the dissolved solute in aqueous TMU solutions at various compositions were recorded respectively by using UV-Visible absorption spectrophotometer (Model UV-2450, Shimadzu) and Fluorolog spectrometer (Jobin-Yvon, Horiba) at controlled temperatures. Fluorescence emission was collected after adjusting the absorbance of the solutions at $\sim 0.1$ with the excitation wavelength fixed at $409 \mathrm{~nm}$. Spectra were then processed by following the standard protocol ${ }^{18,37-40}$ to obtain the spectral frequencies and widths [full-width-at-half-maxima (FWHM)].

The time-correlated single photon counting (TCSPC) technique based on a laser system (Lifespec-ps, Edinburgh, U.K.) with a light-emitting diode (LED) producing excitation at $409 \mathrm{~nm}$ was employed to collect the necessary sets of fluorescence emission intensity decays. The FWHM of the instrument response function (IRF, measured by using water) was found to be $\sim 90$ ps. The sample cuvette was placed into a temperature controlled chamber. For dynamic anisotropy measurements, emission decays were collected at the peak wavelengths of the steady-state emission spectra at three different positions of emission polarizer, magic angle $\left(54.7^{\circ}\right)$, parallel $\left(0^{\circ}, I_{\|}(t)\right)$ and perpendicular $\left(90^{\circ}, I_{\perp}(t)\right)$ with respect to the polarization of the 
excitation light $\left(\lambda_{\text {exc. }}=409 \mathrm{~nm}\right)$. Subsequently, rotational anisotropy decay, $r(\mathrm{t})$, was constructed using the collected decays as follows, ${ }^{41,42}$

$$
r(t)=\frac{I_{\|}(t)-G_{\perp}(t)}{I_{\|}(t)+2 G I_{\perp}(t)},
$$

where, the geometric factor $G$ accounts for the differential sensitivity to the two polarizations which was obtained by tail matching the intensity decays $I_{\|}(t)$ and $I_{\perp}(t)$, and found to be $1.15 \pm 0.15$. The average rotation time for the solute $\left(\left\langle\tau_{r}\right\rangle\right)$ was then obtained via multi-exponential fit to the $\mathrm{r}(\mathrm{t})$ as follows: ${ }^{41,42}\left\langle\tau_{r}\right\rangle=$ $\int_{0}^{\infty} \mathrm{dt}\left[\mathrm{r}(\mathrm{t}) / \mathrm{r}_{0}\right]=\int_{0}^{\infty} \mathrm{dt} \sum_{i=1}^{\mathrm{n}} \mathrm{a}_{\mathrm{i}} \exp \left[-\mathrm{t} / \tau_{i}\right]$, with $\sum_{i=1}^{\mathrm{n}} \mathrm{a}_{\mathrm{i}}=1$ and the initial anisotropy, $\mathrm{r}_{0}=0.376$.

\section{Results and Discussion}

\subsection{Steady State Spectroscopic Studies: Unusual Spectral Behaviour}

Absorption and steady state fluorescence emission spectra of $\mathrm{C} 153$ have been recorded at various binary mixture compositions in the temperature range, $\mathrm{T}$ $\sim 278-353 \mathrm{~K}$, and an anomalous TMU mole fraction $\left(X_{T M U}\right)$ dependence for absorption spectral shift has been observed. Figure 1 presents the absorption and emission spectra of $\mathrm{C} 153$ at a few representative $X_{T M U}$ and $\mathrm{T} \sim 293 \mathrm{~K}$, showing the anomalous composition dependence of the spectral shift. Upper panel of this figure indicates that, similar to aqueous alcohol solutions, ${ }^{17,18,20}$ TMU/water mixtures also exhibit absorption spectral red-shift at low $X_{T M U}$ (up to $X_{T M U} \sim 0.07$ ). Upon further increase of $X_{T M U}$, absorption spectrum shifts to higher frequency (blue-shift, middle panel). Lower panel shows the gradual blueshift of emission spectrum with $X_{T M U} . X_{T M U}$ dependent absorption and emission spectral frequency $(v)$ and width $(\Gamma)$ are shown in figure 2 . Note the total redshift in absorption frequency relative to pure water is $\sim 500 \mathrm{~cm}^{-1}$ (shown by the horizontal dashed lines) that accompanies a spectral narrowing of $\sim 1200 \mathrm{~cm}^{-1}$.

We mentioned the absorption spectral red-shift as anomalous because addition of TMU (static dielectric constant, $\left.\varepsilon_{0} \approx 23\right)^{43}$ into water $\left(\varepsilon_{0} \approx 80\right)$ reduces the average polarity of the medium and thus the extra stabilization of the fluorophore $\mathrm{C} 153$ at low $X_{T M U}$ (reflected by the red-shift) cannot arise from the increased solution polarity. Note here that computer simulation study of TMU/water binary mixtures ${ }^{6}$ suggests enhancement of solution structure. The $\mathrm{H}$-bonding interaction of water-water and water-TMU has been found to
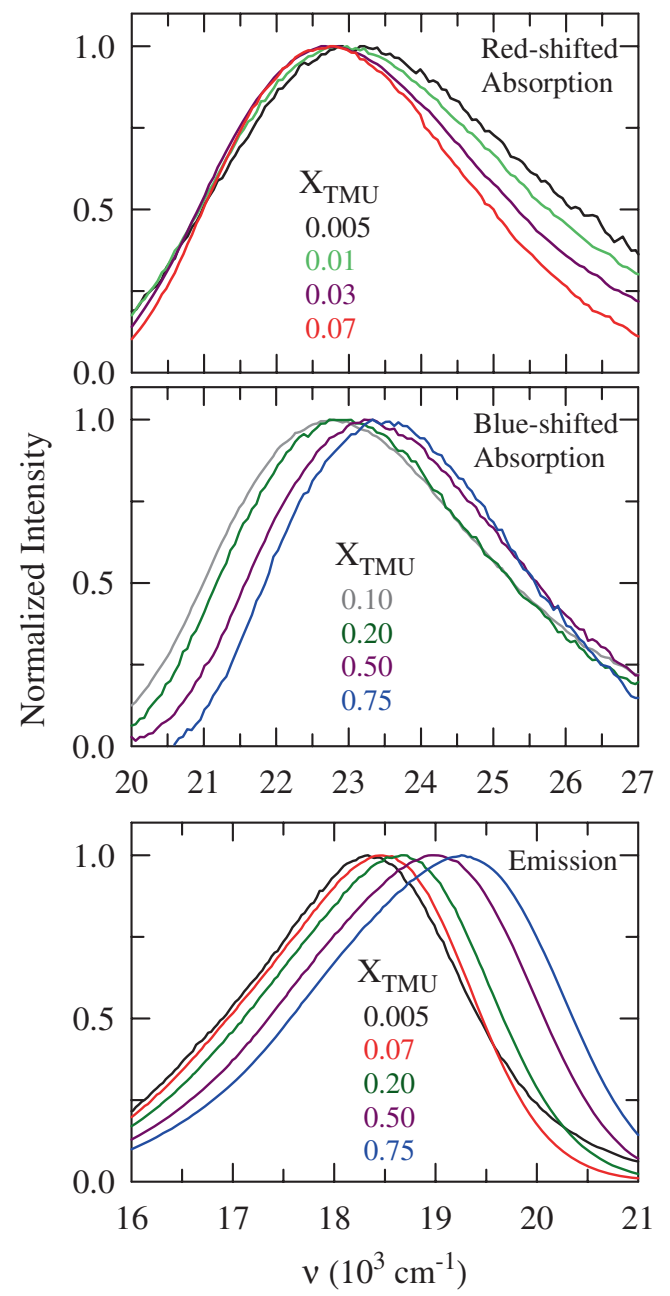

Figure 1. Absorption (upper and middle panels) and emission (lower) spectra of C153 at different TMU mole fractions, $X_{T M U}$ into water at $\mathrm{T}=293 \mathrm{~K}$. Each $X_{T M U}$ is colourcoded and represented in the figure.

be increased upon addition of TMU in water. This enhanced $\mathrm{H}$-bonding leads to more compact or stiffened solution structure ${ }^{17,18}$ leading to the lowering of absorption frequency at low TMU concentrations. However, simulation study ${ }^{6}$ shows that the average H-bond persistence time between two $\mathrm{H}$-bonding specie in this system is in the order of sub-hundred picoseconds. Consequently, the excited state of $\mathrm{C} 153$ which has a lifetime $\sim 2-4$ ns in these solutions experiences a large number of environmental fluctuations, forcing the emission energy to sense only the average solution polarity. As a result, fluorescence emission does not carry any signature of the local solution structure and exhibits a mere dependence on composition dependent solution polarity.

The TMU-induced structural transition may be understood as follows. In the pure state, four coordinated three-dimensional $\mathrm{H}$-bond network structure is the most stable structure of liquid water. When 

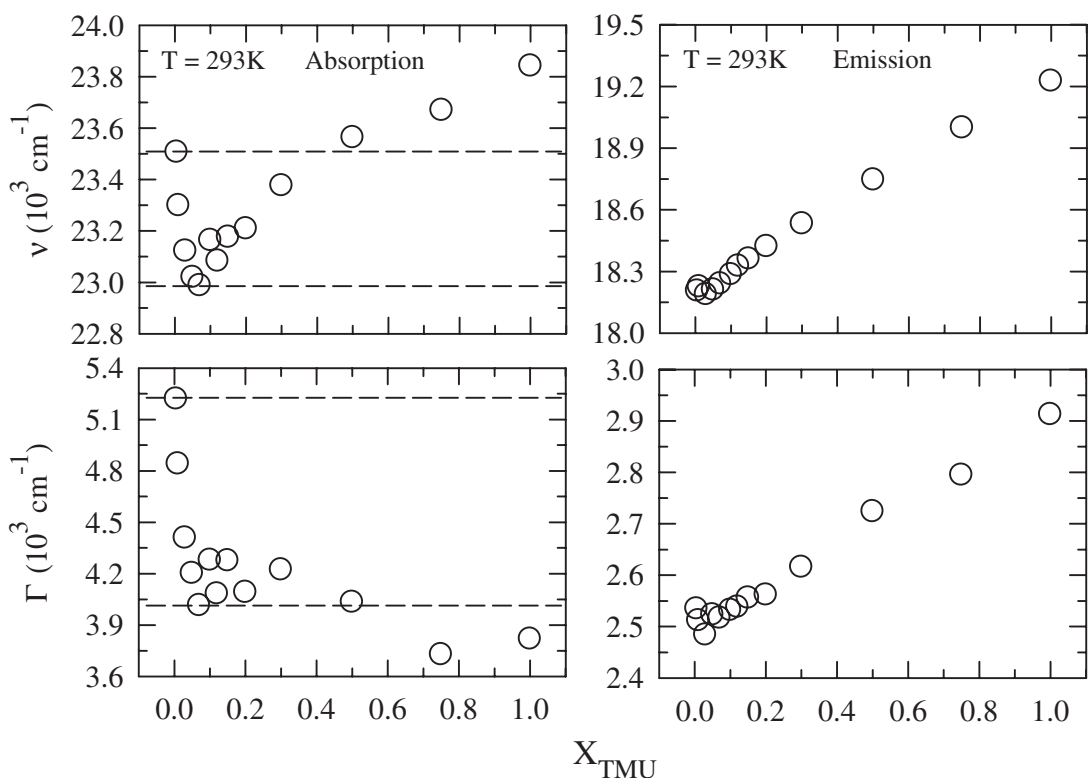

Figure 2. Variation of absorption and emission spectral frequency, $v$ (upper panels) and width, FWHM, $\Gamma$ (lower panels) of C153 with increase of $X_{T M U}$ into water at $\mathrm{T}=293 \mathrm{~K}$. Dashed lines represent red-shift of absorption spectral $v$ and $\Gamma$ at lower $X_{T M U}$. Here, $v$ is obtained by averaging over first moment frequency, peak frequency and half average frequency.

small amount of TMU is added into water, the three dimensional tetrahedral H-bonded network of water can accommodate few TMU molecules. This way the tetrahedral structure of water is retained but a strain is developed in the H-bond network due to nonavailability of certain configurational freedom. ${ }^{44}$ This reduces the compressibility of the medium. ${ }^{45}$ The redshift in absorption spectrum of $\mathrm{C} 153$ reflects this phenomenon. With further addition of TMU into water tetrahedral network of water can no longer accommodate the TMU molecules and it breaks down into two dimensional zigzag chain-like structure. This is shown schematically (scheme 2) below.

We have also noticed by comparing the present results with our previous studies of alcohol/water binary

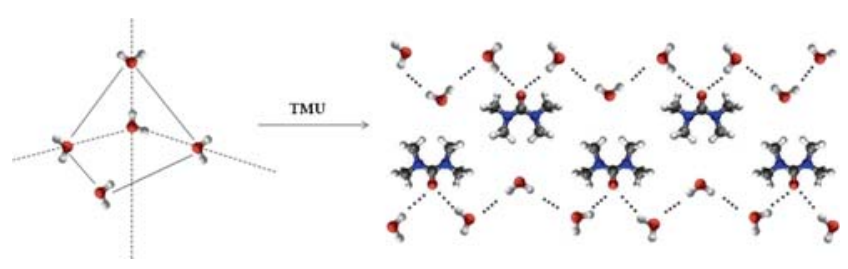

Scheme 2. Cosolvent induced transition from three dimensional H-bond water network to two dimensional zigzag alcohol-like chain structure. mixtures $^{18,20}$ that the extent of red-shift in absorption spectrum is highly dependent on the bulkiness of the hydrophobic moiety present in the molecule. The observed red-shift for TBA/ $\mathrm{H}_{2} \mathrm{O}$ binary mixture was $\sim 1000 \mathrm{~cm}^{-1}$, while it was $\sim 500 \mathrm{~cm}^{-1}$ for $\mathrm{EtOH} / \mathrm{H}_{2} \mathrm{O}$ and 2-butoxyethanol (BE) $/ \mathrm{H}_{2} \mathrm{O}$ mixtures. Absorption and emission spectral widths which have been shown in the lower panels of figure 2 behave in a manner similar to that observed in aqueous solutions of alcohols. ${ }^{17,18,20}$ The absorption narrowing $\left(\sim 1200 \mathrm{~cm}^{-1}\right)$ and emission broadening $\left(\sim 400 \mathrm{~cm}^{-1}\right)$ of spectral widths with $X_{T M U}$ are characteristics of the binary mixtures due to complex medium effects, although the reverse is witnessed in neat liquids. ${ }^{37}$

In figure 3 , excitation wavelength $\left(\lambda_{e x}\right)$ dependence of emission of $\mathrm{C} 153$ in this binary mixture has been monitored at low TMU concentrations where abnormality was found in absorption spectral behaviour. Excitation wavelength dependent emission study is informative where local heterogeneity develops even at ambient condition due to competing interactions in a variety of systems, for example, deep eutectic solvents, ionic liquids, etc. ${ }^{46-54}$ In viscous media, emission spectrum shifts to lower frequency on moving towards longer excitation wavelengths. This phenomenon is known as red-edge-effect (REE). ${ }^{55-57}$ Although, here the viscosity of the medium is very low, ${ }^{58}$ the most dilute TMU solution, $X_{T M U}=0.005$ 


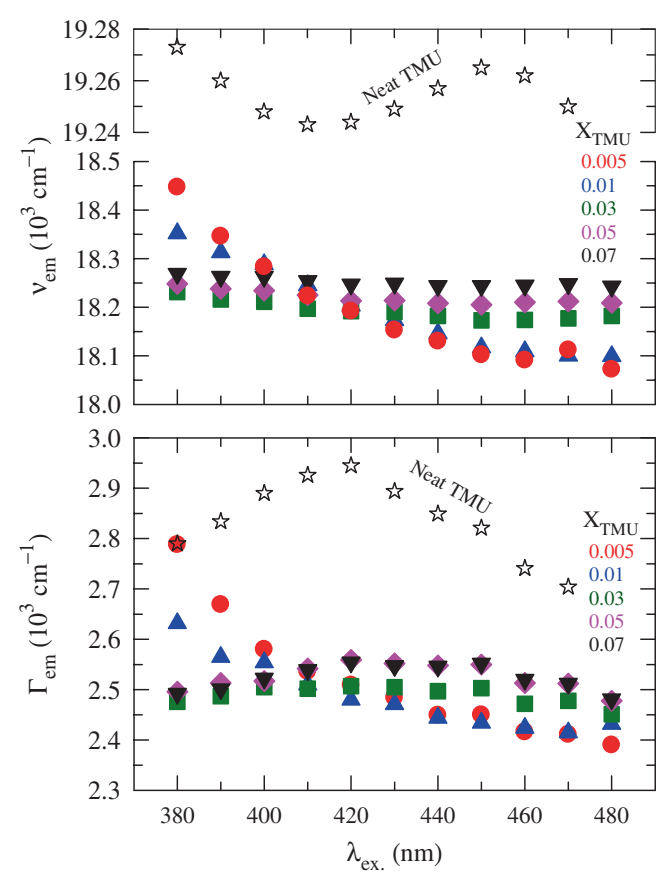

Figure 3. Excitation wavelength $\left(\lambda_{e x}\right)$ dependent emission spectral frequency, $v_{e m}$ (upper panel) and $\Gamma_{e m}$ (lower panel) of C153 at various TMU mole fractions at $\mathrm{T}=293 \mathrm{~K}$. Representation of each TMU mole fraction is colour-coded.

exhibits the maximum $\lambda_{e x}$-dependent shift, $\Delta v_{e m}\left(\lambda_{e x .}\right)$ $=v_{e m}(380 \mathrm{~nm})-v_{e m}(480 \mathrm{~nm}) \sim 400 \mathrm{~cm}^{-1}$, whereas, neat TMU shows $\Delta v_{e m}\left(\lambda_{e x .}\right)=40 \mathrm{~cm}^{-1}$ (upper panel). Further increase of $X_{T M U}$ reduces the $\Delta v_{e m}\left(\lambda_{e x}\right)$. Lower panel shows the similar $\lambda_{e x}$-dependence on $\Gamma_{e m}$. This suggests that solution with $X_{T M U} \sim 0.005$ is the most spatially heterogeneous among the TMU mole fractions studied here. Support of such a view can be accessed from simulation studies which suggest that aggregation of TMU molecules in aqueous mixtures takes place at low TMU concentrations. ${ }^{4-6}$ It also suggests that at $X_{T M U}=0.005$ the aggregated domains are sufficiently longer-lived to be detected via the excitation wavelength dependent study.

\subsection{Search for Temperature Maximum of Absorption Red-shift}

Next, we have investigated the temperature effects on the absorption and emission spectra. In figure 4, temperature dependent total absorption spectral red-shift is shown. The red-shift grows upon increasing temperature from $278 \mathrm{~K}$. It reaches maximum at $\mathrm{T}=$ $293 \mathrm{~K}$. Then, red-shift decreases gradually with further increase of temperature.

Previous studies have also found that the temperature-assisted aggregation due to hydrophobic

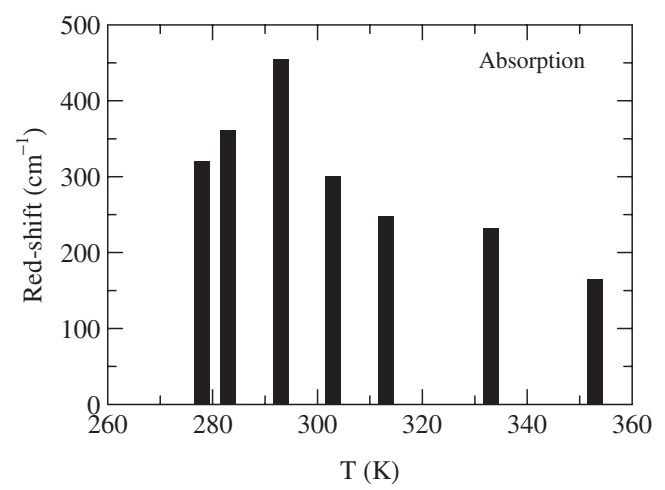

Figure 4. Temperature dependent absorption spectral redshift of $\mathrm{C} 153$ at low TMU concentrations.

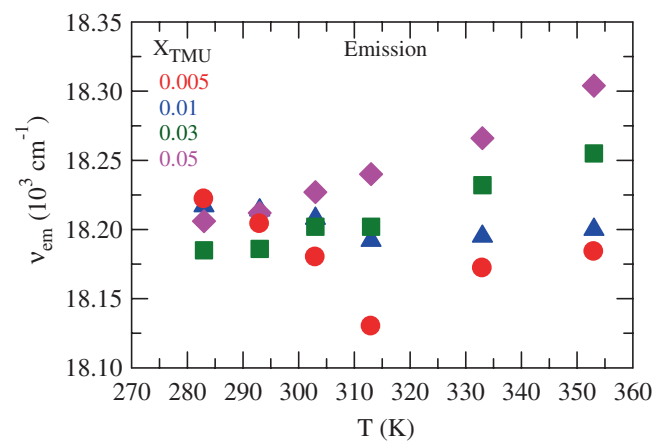

Figure 5. Temperature dependence of emission spectral frequencies at a few representatives TMU mole fractions in the dilute limit.

interaction of small nonpolar solutes and amphiphilic molecules in dilute aqueous solutions. ${ }^{19,59-63}$ Here, the growth of absorption spectral red-shift up to $293 \mathrm{~K}$ may suggest an enhancement of the hydrophobic interaction among the $-\mathrm{N}$ methyl groups of TMU leading to the aggregation of TMU molecules in aqueous solutions at low concentrations. ${ }^{4-6}$ Largest extent of red-shift at $\mathrm{T}=293 \mathrm{~K}$ may therefore indicate maximization of hydrophobic interaction among methyl groups at this temperature. In aqueous solution of TBA, small-angle neutron scattering (SANS) study showed that TBA clustering reaches the maximum for $X_{T B A}=0.04$ at $\sim 353 \mathrm{~K} .{ }^{16}$ The fluorescence spectroscopic study of TBA/water system, ${ }^{19}$ in contrast, could not capture the temperature maximum probably due to the presence of the third component $\mathrm{C} 153$ for reasons suggested for the ternary system of cyclohexene/TBA/water. ${ }^{64} \mathrm{In}$ figure 5, the change of emission frequency of C153 in dilute TMU solutions with temperature displays only blue-shift, reflecting the effects of lowering of average solution polarity with temperature. ${ }^{19}$ 
Table 1. Bi-exponential fit parameters for magic angle fluorescence emission decays and average fluorescence lifetimes $\left(\left\langle\tau_{\text {life }}\right\rangle=\left[a_{1} \tau_{1}+a_{2} \tau_{2}\right] /\left[a_{1}+a_{2}\right] ; a_{1}+a_{2}=1\right)$ of $\mathrm{C} 153$ at various TMU mole fractions at $\mathrm{T}=293 \mathrm{~K}$.

\begin{tabular}{lccccc}
\hline $\mathrm{X}_{\mathrm{TMU}}$ & $a_{1}$ & $\tau_{1} / \mathrm{ns}$ & $a_{2}$ & $\tau_{2} / \mathrm{ps}$ & $\left\langle\tau_{\text {life }}\right\rangle / \mathrm{ns}$ \\
\hline 0.005 & 0.70 & 2.124 & 0.30 & 181 & 1.541 \\
0.01 & 0.91 & 2.230 & 0.09 & 707 & 2.093 \\
0.03 & 0.87 & 2.540 & 0.13 & 345 & 2.255 \\
0.05 & 0.78 & 2.743 & 0.22 & 95 & 2.160 \\
0.07 & 0.86 & 2.897 & 0.14 & 115 & 2.508 \\
0.10 & 0.83 & 3.070 & 0.17 & 106 & 2.566 \\
0.12 & 0.83 & 3.182 & 0.17 & 92 & 2.657 \\
0.15 & 0.85 & 3.344 & 0.15 & 313 & 2.889 \\
0.20 & 0.89 & 3.593 & 0.11 & 455 & 3.248 \\
0.30 & 0.79 & 3.994 & 0.21 & 197 & 3.197 \\
0.50 & 0.77 & 4.617 & 0.23 & 192 & 3.599 \\
0.75 & 0.66 & 5.076 & 0.34 & 79 & 3.377 \\
1.00 & 0.84 & 5.312 & 0.16 & 116 & 4.481 \\
\hline
\end{tabular}

\subsection{Time-resolved Fluorescence Emission Studies}

The average excited-state lifetime $\left\langle\tau_{\text {life }}\right\rangle$ of $\mathrm{C} 153$ at different compositions of TMU into water has been obtained by fitting the magic angle $\left(54.7^{\circ}\right)$ fluorescence emission decay with bi-exponential functions (best numerical representation) at all the TMU concentrations studied here. The fitting parameters are summarized in table 1. A careful observation of these time constants reveals that the long time constant $\tau_{1}$ represents the excited-state lifetime of $\mathrm{C} 153$ whereas the short time constant $\tau_{2}$ may be ascribed to solvent reorganization ${ }^{18}$ although components with faster timescales have been missed due to limited time resolution employed. ${ }^{37}$ While ascribing $\tau_{2}$ exclusively to solvent reorganization it should be noted that such a timescale may also arise due to formation of dye aggregates in solution. ${ }^{65-69}$ The upper and middle panels of figure 6 depict the $X_{T M U}$ dependence of the amplitude-weighted long and short time constants (that is, $a_{i} \tau_{i}$ ), respectively. Both the amplitudeweighted long $\left(a_{1} \tau_{1}\right)$ and short $\left(a_{2} \tau_{2}\right)$ time constants describe different slopes (represented by the dashed lines) depending upon the range of TMU mole fractions. The intersection of two slopes for $a_{1} \tau_{1}$ occurs at $X_{T M U} \sim 0.20$ while that for $a_{2} \tau_{2}$ occurs at $X_{T M U} \sim$ 0.10 . These two different mole fractions may represent structural compactness due to H-bond stiffening in the local solvation shell at the lower mole fraction (picked up by the faster of the two decay components) and over-all structural change at the higher $X_{T M U}$ where, much like in alcohol/water systems, three dimensional H-bond network of water gives way for the two dimensional solution structure after a certain mixture composition. Note also that the slope change for $a_{2} \tau_{2}$ occurs
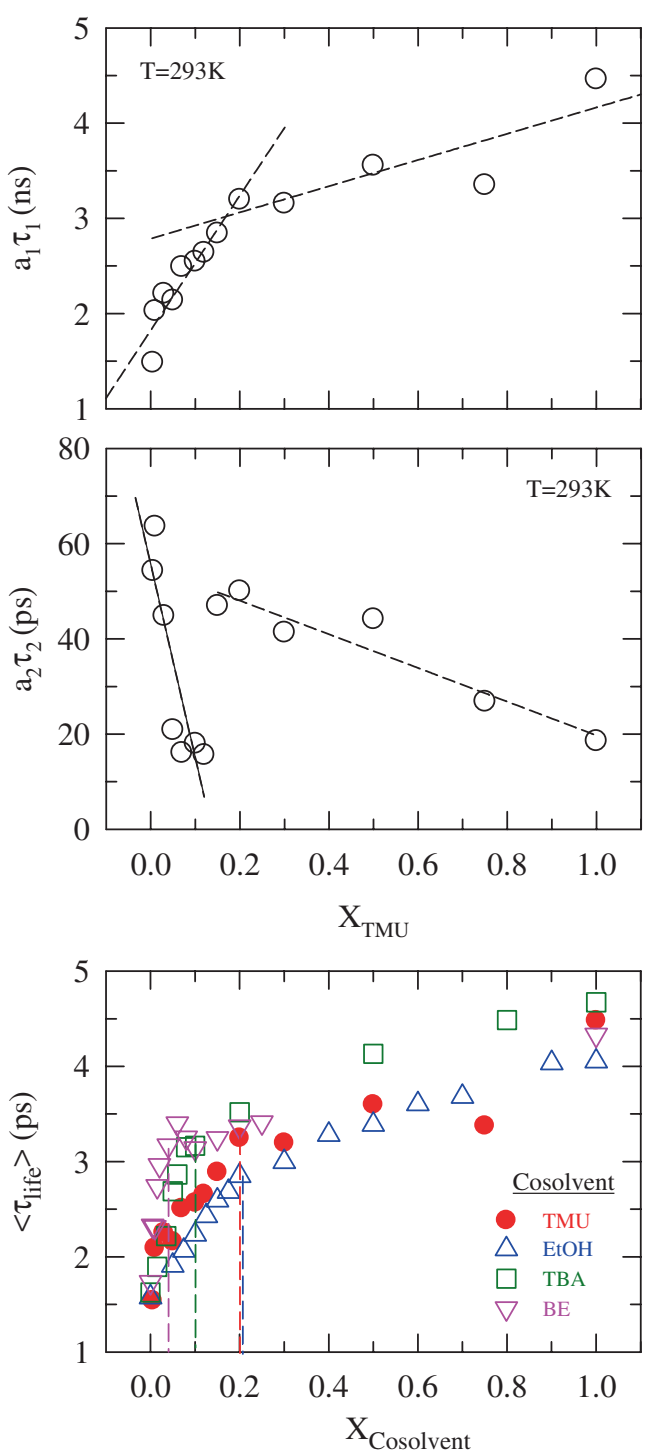

Figure 6. $X_{T M U}$-dependence of the time constants (amplitude weighted) for bi-exponential fit of excited state fluorescence lifetime decay (upper and middle panels) and average life $\left\langle\tau_{\text {life }}\right\rangle$ of C153 (lower panel) at $\mathrm{T}=293 \mathrm{~K}$. For comparison $\left\langle\tau_{\text {life }}\right\rangle$ of $\mathrm{C} 153$ in $\mathrm{EtOH}+$ Water, TBA+Water and $\mathrm{BE}+$ Water binary mixtures are also plotted in lower panel. Dashed lines in the lower panel are shown to indicate the cosolvent mole fraction at which slope changes due to structural transition.

at a TMU mole fraction very close to that $\left(X_{T M U} \sim\right.$ $0.07)$ where the largest absorption red-shift has been recorded.

In the lower panel of the figure we show the composition dependent $\left\langle\tau_{\text {life }}\right\rangle$ of C153 in TMU-water solutions and compared with those measured for the other three different aqueous alcohol (EtOH, TBA, BE) solutions. ${ }^{18,20}$ We observe very similar behaviour for TMU/water and EtOH/water binary mixtures. Both the binary mixtures encounter the over-all solution structural transition at $X_{T M U, E t O H}=0.20$, whereas the 
Table 2. Static dielectric constant $\left(\varepsilon_{0}\right)$ and radius $(r)$ of four different cosolvents of water.

\begin{tabular}{lcc}
\hline Cosolvent & $\varepsilon_{0}$ & $r(\AA)$ \\
\hline EtOH & 24 & 2.33 \\
TMU & 23 & 3.50 \\
TBA & 12 & 2.76 \\
BE & 9 & 3.15 \\
\hline
\end{tabular}

transition occurs for TBA/water and $\mathrm{BE} /$ water solutions at $X_{T B A}=0.10$ and $X_{B E}=0.04$ respectively. Scheme 1 and table $2^{43,70-74}$ indicate that size of TMU is greater than that of $\mathrm{EtOH}$ as the former contains larger hydrophobic moieties, although they possess nearly equal solvent polarity. Table 2 also indicates that presence of cosolvent with relatively less polarity (as represented by $\varepsilon_{0}$ ) shifts the transition mole fraction to lower cosolvent concentration. This suggests that besides the size of the hydrophobic groups, polarity of the cosolvent also plays an important role in determining the transition composition.

We have further explored how the rotation of the solute $\mathrm{C} 153$ gets affected in this binary mixture. Note single exponential function of time was required for adequate fitting of $r(t)$ decays at very low TMU concentrations and pure components, whereas $r(t)$ decays at other higher TMU concentrations were fitted by double exponential function. All the fit parameters along with the average rotation time $\left\langle\tau_{r}\right\rangle$ are provided in table 3 . Note that these well separated two time constants arise due to the inherent nature of the underlying time-dependent friction. ${ }^{41}$ Representative $r(t)$ decay and corresponding residual of $\mathrm{C} 153$ in $X_{T M U}=0.05$ at $\mathrm{T}=293 \mathrm{~K}$ are shown in figure 7 . Here, the residual reflects the goodness of bi-exponential fitting.

Table 3. Fit parameters for $r(t)$ decays and average rotational time, $\left\langle\tau_{r}\right\rangle$ of $\mathrm{C} 153$ at various concentrations of TMU in water at $\sim 293 \mathrm{~K}$.

\begin{tabular}{lccccc}
\hline $\mathrm{X}_{\mathrm{TMU}}$ & $a_{1}$ & $\tau_{1} / \mathrm{ps}$ & $a_{2}$ & $\tau_{2} / \mathrm{ps}$ & $\left\langle\tau_{r}\right\rangle / \mathrm{ps}$ \\
\hline 0.005 & 1.00 & 53 & & & 53 \\
0.01 & 1.00 & 69 & & & 69 \\
0.03 & 1.00 & 98 & & & 98 \\
0.05 & 0.52 & 227 & 0.48 & 33 & 133 \\
0.07 & 0.51 & 279 & 0.49 & 37 & 159 \\
0.10 & 0.58 & 315 & 0.42 & 29 & 194 \\
0.12 & 0.58 & 368 & 0.42 & 33 & 229 \\
0.15 & 0.65 & 346 & 0.35 & 26 & 234 \\
0.20 & 0.64 & 399 & 0.36 & 19 & 263 \\
0.30 & 0.54 & 389 & 0.46 & 32 & 223 \\
0.50 & 0.53 & 248 & 0.47 & 24 & 143 \\
0.75 & 0.38 & 173 & 0.62 & 28 & 83 \\
1.00 & 1.00 & 59 & & & 59 \\
\hline
\end{tabular}

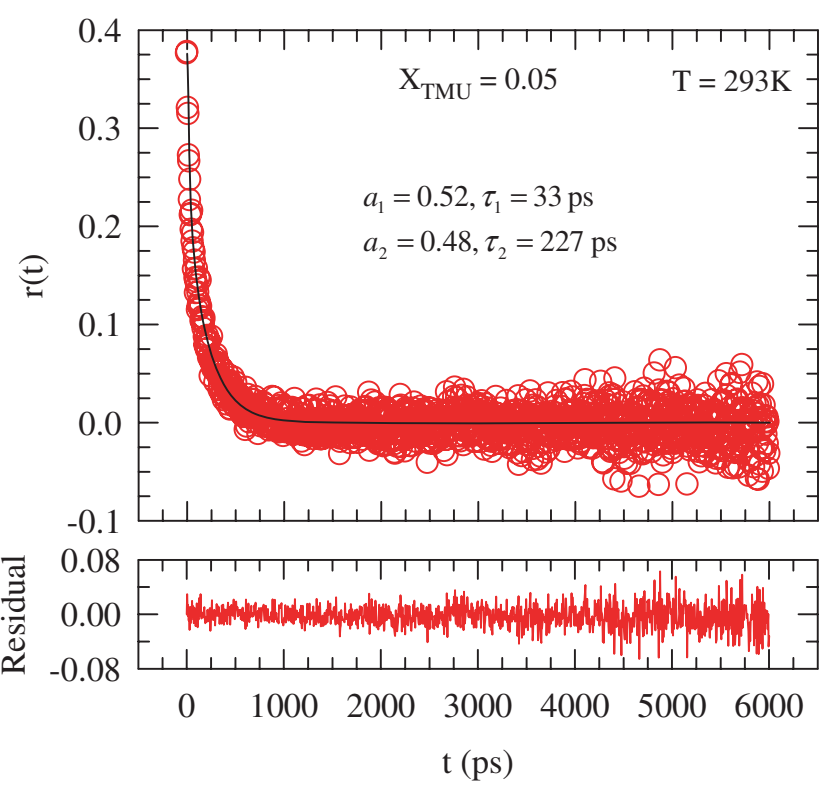

Figure 7. Rotational anisotropy decay, $r(t)$ (red circles, upper panel) of C153 with bi-exponential fitting (solid black line, upper panel) in $X_{T M U}=0.05$ at $\sim 293 \mathrm{~K}$ and corresponding residual of the fit (lower panel). Fitting parameters are also summarized in the inset of upper panel.

Figure 8 depicts the composition dependence of $\left\langle\tau_{r}\right\rangle$ in TMU/water mixtures, and compares with the data obtained for $\mathrm{C} 153$ in aqueous solutions of $\mathrm{EtOH},{ }^{18}$ $\mathrm{TBA}^{18}$ and BE. ${ }^{20}$ An interesting aspect of this figure is that $\left\langle\tau_{r}\right\rangle$ increases gradually with increase of $X_{T M U}$ up to $X_{T M U}=0.20$. Then $\left\langle\tau_{r}\right\rangle$ abruptly changes its slope and decreases continuously up to $X_{T M U}=1$. Here also the slope change of $\left\langle\tau_{r}\right\rangle$ takes place at the same concentration $\left(X_{T M U}=0.20\right)$ where a reversal of the slope for $\left\langle\tau_{\text {life }}\right\rangle$ took place.

The rotational behaviour of $\mathrm{C} 153$ at various $X_{T M U}$ correlates well with the viscosity of the aqueous TMU

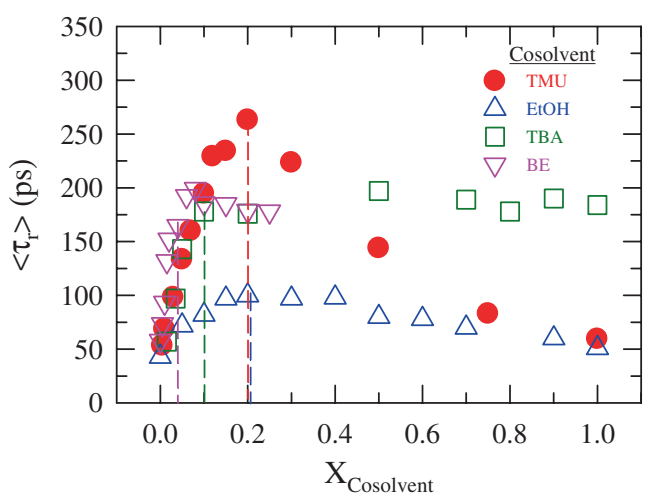

Figure 8. Variation of average rotational time, $\left\langle\tau_{r}\right\rangle$ of C153 with increase of cosolvent concentration into water, $X_{\text {Cosolvent }}$. Dashed lines are shown to indicate the $X_{\text {Cosolvent }}$ where solution structural transition takes place. Symbolic representations of cosolvents are given in the figure. 


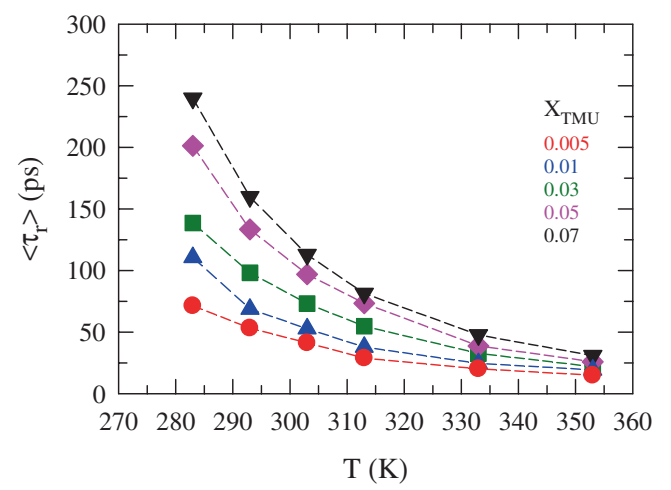

Figure 9. Temperature dependence of $\left\langle\tau_{r}\right\rangle$ of $\mathrm{C} 153$ at various TMU mole fractions, $X_{T M U}$.

solutions. ${ }^{58}$ Including viscosity other physicochemical properties of this binary mixture, such as, density, ultrasonic absorption, sound velocity have been also found to attain the maximum at $X_{T M U}=0.20 .^{58,75}$ Although the concept of formation of "tetramethylurea tetrahydrate" at $X_{T M U}=0.20$ prevailed for sometimes, ${ }^{75}$ subsequent computer simulation study ruled out that possibility. ${ }^{76}$ By comparing the $\left\langle\tau_{r}\right\rangle$ values of $\mathrm{C} 153$ at various compositions of other cosolvents $(\mathrm{EtOH}$, TBA, BE) in water, we observed that in the presence of either of the cosolvent TMU or EtOH, aqueous solutions undergo solution structural transition at equivalent composition of the cosolvent $\left(X_{T M U, E t O H}=0.20\right)$. The decreasing order of $X_{\text {cosolvent }}$, at which the transition occurs, is $X_{T M U} \approx X_{E t O H}>X_{T B A}>X_{B E}$. The maximum slowing down at lower mole fractions of the cosolvents has been observed for TMU/water binary mixture. This indicates that TMU induces comparatively more ordering in the structure of the aqueous solutions. Hence, these four $\mathrm{N}-$ methyl groups of TMU acts in the similar way as methyl groups of alcohol molecules do.

We have also explored the temperature effects on the $\left\langle\tau_{r}\right\rangle$ of $\mathrm{C} 153$ at several different TMU concentrations in figure 9, but did not observe any irregularity for any particular TMU concentration. $\left\langle\tau_{r}\right\rangle$ becomes faster with increase of temperature for all the compositions studied here suggesting only the lowering of viscosity of the medium with rise of temperature that solely dictates the solute rotation at a given mixture composition.

\section{Conclusion}

In summary, our present experimental results indicate the hydrophobic nature of $\mathrm{N}$-methyl groups of TMU, ${ }^{1}$ and suggest effects on structure of aqueous solution as those observed for several water/alcohol binary mixtures. Strengthening of H-bond structure of water found in simulation study has been reflected here by the observed red-shift of the absorption spectrum of C153 at the lower concentration of TMU. Excitation wavelength dependence of the emission of $\mathrm{C} 153$ at very low TMU mole fraction also confirms the aggregation of TMU molecules at very dilute condition. ${ }^{4-6}$ Interestingly, we have seen the presence of temperature maximum for hydrophobic interaction induced aggregation in TMU/water binary mixtures, which was observed before via SANS measurements for aqueous solutions of TBA. ${ }^{16}$ By analogy to water/alcohol mixtures, we propose that TMU also induces transition from tetrahedral H-bond network to chain-like $\mathrm{H}$-bond structure upon successive addition of TMU in water. A comparison to observation made earlier for aqueous alcohol mixtures reveals that this transition point depends upon both the size of the hydrophobic (here alkyl groups) moiety attached to the cosolvent molecules and the cosolvent polarity.

\section{Acknowledgments}

S.I. thanks University Grants Commission (UGC), India for a research fellowship.

\section{References}

1. Koga $Y$, Westh $P$, Nishikawa $K$ and Subramanian $S$ 2011 J. Phys. Chem. B 1152995

2. Pace C N and Marshall H F 1980 Arch. Biochem. Biophys. 199270

3. Barone G, del Vecchio P, Giancola C and Graziano G G 1993 Thermochim. Acta 22767

4. Marcus Y 2002 Phys. Chem. Chem. Phys. 44462

5. Almásy L, Len A, Székely N K and Pleštil J 2007 Fluid Phase Equilib. 257114

6. Indra S and Biswas R 2015 Mol. Sim. 41471

7. Franks F and Ives D J G 1966 Q. Rev. Chem. Soc. 201

8. Beddard G S, Doust T and Hudales J 1981 Nature 294 145

9. Brai M and Kaatze U 1992 J. Phys. Chem. 908946

10. Soper A K and Finney J L 1993 Phys. Rev. Lett. 714346

11. Yoshida $\mathrm{K}$ and Yamaguchi $\mathrm{T} 2001$ Z. Naturforsch. 56A 529

12. Dixit S, Crain J, Poon W C K, Finney J L and Soper A K 2002 Nature 416829

13. Murthy S S N 1999 J. Phys. Chem. A 1037927

14. Wojtkow D and Czarnecki M 2005 J. Phys. Chem. A 109 8218

15. Kusalik P G, Lyubertsev A P, Bergman D L and Laaksonen A 2000 J. Phys. Chem. B 1049533

16. Bowron D T and Finney J L 2007 J. Phys. Chem. B 111 9838

17. Pradhan T, Ghoshal $\mathrm{P}$ and Biswas R 2008 J. Phys. Chem. A 112915

18. Pradhan T, Ghoshal P and Biswas R 2008 J. Chem. Sci. 120275

19. Gazi H A R and Biswas R 2011 J. Phys. Chem. A 115 2447 
20. Indra S and Biswas R 2015 J. Chem. Phys. 142204501

21. Koga Y 1996 J. Phys. Chem. 1005172

22. Gupta R and Patey G N 2011 J. Phys. Chem. B 115 15323

23. Pascal T A and Goddard W A 2012 J. Phys. Chem. B 11613905

24. Matvejev V, Zizi M and Stiens J 2012 J. Phys. Chem. B 11614071

25. Koga Y, Nishikawa K and Westh P 2004 J. Phys. Chem. A 1083873

26. Marcus Y 2011 J. Mol. Liq. 15823

27. Roy S, Banerjee S, Biyani N, Jana B and Bagchi B 2011 J. Phys. Chem. B 115685

28. Tielrooij K, Hunger J, Buchner R, Bonn M and Bakker H J 2010 J. Am. Chem. Soc. 13215671

29. Rezus Y L A and Bakker H J 2008 J. Phys. Chem. A 1122355

30. Rezus Y L A and Bakker H J 2007 Phys. Rev. Lett. 99148301

31. Mazur K, Heisler I A and Meech S R 2011 J. Phys. Chem. B 1152563

32. Laage D, Stirnemann G and Hynes J T 2009 J. Phys. Chem. B 1132428

33. Stirnemann G, Sterpone F and Laage D 2011 J. Phys. Chem. B 1153254

34. Brandeburgo W H, van der Post S T, Meijerab E J and Ensing B 2015 Phys. Chem. Chem. Phys. 1724968

35. Hummer G, Rasaiah J C and Noworyta J P 2011 Nature 414188

36. Xu Y, Gnanasekaran R and Leitner D M 2012 J. Atom. Mol. Opt. Phys. 125071

37. Horng M L, Gardecki J A, Papazyan A and Maroncelli M 1995 J. Phys. Chem. B 9917311

38. Biswas R, Lewis J E and Maroncelli M 1999 Chem. Phys. Lett. 310485

39. Lewis J E, Biswas R, Robinson A G and Maroncelli M 2001 J. Phys. Chem. B 1053306

40. Pradhan $\mathrm{T}$ and Biswas R 2007 J. Phys. Chem. A 11111514

41. Horng M L, Gardecki J A and Maroncelli M 1997 J. Phys. Chem. A 1011030

42. Dahl K, Biswas R, Ito N and Maroncelli M 2005 J. Phys. Chem. B 1091563

43. Fratiello A, Lee R E, Miller D P and Nishida V M 1967 Mol. Phys. 13349

44. Chandler D 2005 Nature 437640

45. Omelyan I, Kovalenko A and Hirata F 2003 J. Theor. Comput. Chem. 2193

46. Guchhait B, Gazi H A R, Kashyap H K and Biswas R 2010 J. Phys. Chem. B 1145066

47. Das A and Biswas R 2015 J. Phys. Chem. B 11910102
48. Jin H, Li X and Maroncelli M 2007 J. Phys. Chem. B 11113473

49. Mandal P K, Sarkar M and Samanta A 2004 J. Phys. Chem. A 1089048

50. Das A, Das S and Biswas R 2013 Chem. Phys. Lett. $\mathbf{5 8 1} 47$

51. Das A, Das S and Biswas R 2015 J. Chem. Phys. 142 034505

52. Guchhait B, Das S, Daschakraborty S and Biswas R 2014 J. Chem. Phys. 140104514

53. Guchhait B, Daschakraborty S and Biswas R $2012 \mathrm{~J}$. Chem. Phys. 136174503

54. Pal T and Biswas R 2011 Chem. Phys. Lett. 517180

55. Lakowicz J R 1999 In Principles of Fluorescence Spectroscopy (New York: Kluwer Academic)

56. Galley W C and Purkey R M 1970 Proc. Natl. Acad. Sci. U.S.A. 671116

57. Demchenko A P 2002 Luminescence 1719

58. Arêas E P G, Arêas J A G, Hamburger J, Peticolas W L and Santos P S 1996 J. Colloid Interface Sci. 180578

59. Euliss G W and Sorensen C M 1984 J. Chem. Phys. 804767

60. Bender T M and Pecora R 1988 J. Phys. Chem. 921675

61. Raschke T M, Tsai J and Levitt M 2001 Proc. Natl. Acad. Sci. U.S.A. 985965

62. Paschek D 2004 J. Chem. Phys. 1206674

63. Ito N, Kato T and Fujiyama T 1981 Bull. Chem. Soc. Jpn. 542573

64. Bowron D T and Moreno S D 2005 J. Phys. Chem. B 10916210

65. Cigáň M, Donovalová J, Szöcs V, Gašpar J, Jakusová K and Gáplovský A 2013 J. Phys. Chem. A 1174870

66. Verma P and Pal H 2014 J. Phys. Chem. A 1186950

67. Verma P and Pal H 2013 J. Phys. Chem. A 11712409

68. Verma P and Pal H 2012 J. Phys. Chem. A 1164473

69. Cigáň M, Danko M, Donovalová J, Gašpar J, Stankovičová H, Gáplovský A and Hrdlovič P 2014 Spectrochim. Acta, A $\mathbf{1 2 6} 36$

70. Dutt G B and Doraiswamy S J. Chem. Phys. 962475

71. Dutt G B, Doraiswamy S and Periasamy N $1991 \mathrm{~J}$. Chem. Phys. 945360

72. Douhéret G and Pal A 1988 J. Chem. Eng. Data 3340

73. Biswas R 1998 Ph.D thesis (Indian Institute of Science, Bangalore)

74. www.chemicalize.org

75. Sasaki K and Arakawa K 1973 Bull. Chem. Soc. Jpn. 462738

76. Belletato P, Freitas L C G, Arêas E P G and Santos P S 1999 Phys. Chem. Chem. Phys. 14769 